\title{
La agricultura urbana en la planificación de las ciudades: entre la participación y el mercado
}

\author{
Urban agriculture in the planning of cities: \\ between participation and the market
}

\author{
Marta Soler Montiel ${ }^{*}$ y Henk Renting ${ }^{* *}$
}

Hábitat y Sociedad (ISSN 2173-125X), n. ${ }^{\circ}$, noviembre de 2014, pp. 5-11.

* Universidad de Sevilla.

** Fundación RUAF (Centros de Recursos de Agricultura Urbana y Seguridad Alimentaria).
$\mathrm{E}$ debate académico y público sobre sostenibilidad urbana y urbanismo está prestando una especial atención a la agricultura urbana. Como consecuencia, en algunos países, y en concreto en algunas ciudades, se han comenzado a diseñar políticas urbanísticas con criterios de sostenibilidad que incorporan la agricultura como una actividad a desarrollar en el espacio urbano, destinando suelo específicamente para estas actividades o promoviendo huertos en espacios vacantes dentro de las tramas urbanas consolidadas o en espacios agrícolas del periurbano. A su vez, algunas políticas locales están apoyando el desarrollo de canales cortos de comercialización alimentaria urbana en alianza con la agricultura periurbana, avanzando así hacia la autosuficiencia alimentaria y la mejora ambiental de las ciudades. Sin embargo, ésta no es una tendencia generalizada y en muchas ciudades se carece de una política pública y de una ordenación urbanística que integre la agricultura urbana en el diseño de la ciudad.

Por otra parte, las motivaciones que impulsan y explican las políticas públicas de agricultura urbana son muy diversas según los agentes sociales y políticos implicados. A menudo es la ciudadanía quien reclama e impulsa el retorno y la defensa de la agricultura en las ciudades impulsando el diseño de nuevas políticas urbanas que atienden estos reclamos sociales. Pero también, en un contexto de urbanismo neoliberal y de crisis fiscal de los municipios, las Administraciones locales pueden encontrar en las demandas de participación, de espacios verdes de ocio y de espacios productivos alimentarios una oportunidad para externalizar y privatizar servicios sociales públicos que pasan a ser asumidos por la sociedad civil sin recursos materiales ni humanos adecuados para ello. Consecuentemente, en la actualidad, la agricultura urbana se desenvuelve inmersa en fuertes tensiones entre la participación social que reclama la ciudadanía, las exigencias del mercado que presiona los usos del suelo urbano y las políticas urbanísticas locales que median entre ambas. Este número monográfico se centra en el análisis de las políticas públicas locales que encuentran en la agricultura urbana un instrumento para el diseño de las ciudades a través de distintas estrategias de planificación e intervención.

Este número monográfico se abre con la reimpresión del artículo de Marit Rosol, profesora del Departamento de Geografía Humana de la Universidad Goethe de Frankfurt en Alemania, titulado "Public Participation in Post-Fordist Urban Green Space Governance: The case of 
Community Gardens in Berlin” publicado en septiembre de 2010 en la revista International Journal of Urban and Regional Research. El trabajo de campo que sustenta este artículo se centra en cuatro 'jardines comunitarios', definidos como espacios verdes urbanos públicos gestionados por la ciudadanía, en Berlín; cuya gestión y estructura coinciden en gran parte con los huertos comunitarios en espacios verdes públicos de distintas ciudades europeas.

Dos de los proyectos estudiados se iniciaron en la década de los ochenta impulsados por grupos vecinales con fuerte vinculación con los nuevos movimientos sociales urbanos, como la okupación, caracterizados por la confrontación con las autoridades locales. Los otros dos proyectos se inician en torno al año 2000 con el apoyo e incluso el impulso de la Administración local. El artículo argumenta que esta evolución refleja un cambio en el papel del Estado que abandona progresivamente su rol de proveedor de servicios públicos, propio del Estado del Bienestar, para avanzar hacia un Estado neoliberal que externaliza y privatiza servicios públicos, en este caso, el mantenimiento de espacios verdes urbanos. Este nuevo Estado neoliberal encuentra en el voluntariado ciudadano una herramienta eficaz y a bajo coste para mantener la provisión de ciertos servicios sociales sin asumir ni el coste ni la responsabilidad sobre los mismos.

El análisis no plantea, sin embargo, una realidad dicotómica ni una evolución lineal sino que muestra una situación compleja en la que en los cuatro proyectos aparecen elementos de participación y dinamismo social, así como de negociación y colaboración con las autoridades locales. Por una parte, se evidencia cómo el creciente interés de la Administración local de Berlín por los jardines comunitarios, hasta llegar a promoverlos, es el resultado de un nuevo modelo neoliberal en el Gobierno urbano que genera una reducción de fondos públicos. En este contexto, la participación ciudadana se ve como una estrategia para solucionar esta situación de forma circunstancial, ya que los jardines comunitarios son vistos por la Administración como "actividades intermedias" a la espera de que el mercado reclame usos alternativos para estos suelos urbanos. Consecuentemente, estos espacios se caracterizan por una elevada fragilidad en ciudades neoliberales con una visión instrumentalista de la participación ciudadana.

A la vez, los jardines comunitarios analizados se convierten en espacios sociales vivos de encuentro de la comunidad, donde tienen lugar intensos procesos de participación ciudadana, generando vínculos a través de actividades sociales y medioambientales gestionadas de forma comunitaria. En estos espacios se produce, pues, una verdadera y profunda construcción participativa de la ciudad, más allá de los fines de la Administración local que los alberga. Se genera así una situación compleja y contradictoria donde a la vez que se sirve a estrategias y fines neoliberales se avanza en la senda de la emancipación. Marit Rosol concluye su análisis defendiendo un enfoque analítico de los procesos participativos en contraste con un enfoque normativo, recordando que las realidades urbanas son muy diversas y dependen de las coyunturas específicas de cada ciudad, así como de su tradición histórica y de los contextos legislativos y socioeconómicos nacionales en que se desenvuelven. Este artículo, además de ser una estimulante invitación para continuar realizando investigaciones situadas de los procesos participativos que están impulsando la agricultura urbana y la gestión comunitaria de espacios públicos verdes en las ciudades de todo el mundo, aporta agudas reflexiones sobre las contradiccio- 
nes y complejidades de las políticas urbanas orientadas a promover la agricultura urbana de forma participativa en un contexto de globalización neoliberal.

Nerea Morán, arquitecta urbanista e investigadora del Grupo de Investigación en Urbanismo, Arquitectura y Sostenibilidad (GIAU+S) de la Universidad Politécnica de Madrid y José Luis Casadevante, sociológo integrante de la cooperativa Garúa, son los autores del siguiente artículo titulado "A desalambrar. Agricultura urbana, huertos comunitarios y regulación urbanística”. Este trabajo se centra en el análisis comparado de la agricultura urbana en tres ciudades, Londres, Berlín y San Francisco, en las que esta actividad tiene una larga trayectoria y se desarrolla en la actualidad en contextos normativos diversos que la dotan de estabilidad. Los marcos legislativos y las estrategias de institucionalización de la agricultura urbana en los casos señalados se comparan con la situación del Estado español donde se carece de un marco regulatorio que permita integrar en la planificación urbanística esta actividad.

El estudio diferencia entre huertos tradicionales y huertos comunitarios, siendo estos últimos los analizados por Marit Rosol en Berlín en el caso anterior. Entre los primeros se encuentran los allotments ingleses o los kleingarten o schrebergärten alemanes que en España toman la forma de huertos de autoconsumo. Estos huertos urbanos tienen en el norte de Europa una larga tradición y están, por tanto, ampliamente institucionalizados y regulados, en algunos casos, como en Inglaterra, desde principios del siglo XX. Esta forma de agricultura urbana, que históricamente en Inglaterra y Alemania ha estado vinculada a la industrialización sirviendo para paliar situaciones de hambruna y pobreza, aparece en España más tarde, a partir de la década de los ochenta. Desde entonces han proliferado en distintas ciudades a iniciativa de Ayuntamientos y grupos vecinales para ubicarse prioritariamente en zonas verdes y destinadas a equipamientos con un grado de institucionalización mucho menor del que se dan en los casos noreuropeos analizados.

Estados Unidos es el país pionero en los huertos comunitarios que surgen en la década de los setenta como estrategia de lucha contra la pobreza vinculada a movimientos sociales y vecinales. Desde entonces, en las últimas décadas, se han creado huertos comunitarios en numerosos países de todo el mundo, en algunos casos con orientaciones más hacia el ocio y el disfrute del aire libre y en otras más centrados en el cultivo para la alimentación cotidiana de la gente. El estudio pone de manifiesto que este segundo tipo de agricultura urbana se caracteriza por un menor grado de institucionalización y, consecuentemente, una mayor vulnerabilidad derivada de la situación de cesión o contratos temporales con la que acceden al uso del suelo, mayoritaria pero no exclusivamente, público. Esta situación de temporalidad es especialmente cuestionada por los autores que reconocen el valor de esta medida transitoria, pero que critican la reversibilidad de la medida y, por tanto, la debilidad del compromiso público en el cambio estructural del modelo de ciudad. Este artículo resalta que, mientras que en Inglaterra y Alemania se reconoce legalmente esta forma de agricultura y existe alguna forma de institucionalización, en España la mayoría de los huertos comunitarios se mantienen en la "alegalidad", estando por tanto en una situación de fragilidad extrema. En este sentido, es paradigmática la situación irregular del $80 \%$ de los huertos integrados en la Red de Huertos de Madrid, Red que ha sido 
reconocida como buena práctica por el programa Hábitat de Naciones Unidas y el Ministerio de Fomento.

El análisis comparativo de las formas de regulación de la agricultura urbana en las tres ciudades señaladas pone de manifiesto la diversidad de estrategias legales e institucionales que existen para incorporar esta actividad a la planificación y el diseño de las ciudades, siendo una cuestión fundamental el reconocimiento explícito de la agricultura como uso admisible del suelo urbano, con calificación y categoría propia o al menos como uso permitido en otras categorías de suelo urbano. En el Estado español se carece de todo instrumento de regulación y reconocimiento legal de la agricultura urbana, situación criticada por los autores, que reclaman una ley específica de agricultura urbana en el ámbito estatal, así como cambios en las leyes del suelo autonómicas para poder incorporar la agricultura urbana al planeamiento urbanístico a través de "redes de zonas verdes y espacios libres agrícolas", recomendándose, en última instancia, el diseño de programas municipales de agricultura urbana. Todo un camino por hacer.

A continuación, Markus Schermer, profesor del Departamento de Sociología de la Universidad de Innsbruck en Austria, analiza la contribución a la construcción de una ciudad más sostenible de los huertos interculturales en Innsbruck en el artículo "Transnational at home: intercultural gardens and the social sustainability of cities in Innsbruck, Austria”. De la mano de un estudio de caso, en este artículo se reflexiona sobre los procesos migratorios en la globalización, identificando la agricultura urbana como un espacio idóneo para generar el encuentro multicultural, a la vez que se exploran, tanto desde una perspectiva teórica como analítica, las relaciones entre la agricultura urbana, los procesos migratorios y la sostenibilidad urbana.

Los huertos multiculturales tienen ya cierto arraigo en Alemania y Austria y, aunque frecuentemente parten de una iniciativa ciudadana, encuentran el apoyo institucional de los municipios que facilitan la tierra, el agua y las infraestructuras básicas. Se trata, pues, de un caso de política local de incorporación de la agricultura a la ciudad. En 2008, a propuesta de la sociedad civil y con apoyo municipal, se crea en el sur de la ciudad de Innsbruck el huerto intercultural analizado en el que unas 60 personas de 25 países, incluida población local, siendo dos tercios mujeres, cultivan tanto en bancales individuales, como colectivos y comunales. El huerto cuenta además con espacios comunes para actividades sociales, juegos infantiles y actividades de recreo.

El estudio muestra cómo las motivaciones para cultivar son diversas, múltiples y complejas, combinando la alimentación con la búsqueda de espacios terapéuticos, de encuentro o de ocio. El hecho complejo de cultivar y alimentarse genera un proceso de localización y arraigo que se articula con las experiencias de los nuevos procesos migratorios transnacionales, marcados por las posibilidades de comunicación que ofrecen las nuevas tecnologías, en los que las identidades y las vidas cotidianas de las personas migrantes se construyen a través de múltiples y continuas interconexiones entre espacios en más de un país. En este nuevo contexto, el autor argumenta que los huertos interculturales se configuran como espacios transnacionales multiétnicos localizados. Cultivar es una actividad localizada que conecta a la persona con la tierra, el clima y las estaciones del lugar donde habita, transplantándola y enraizándola de nuevo, uniendo pasado y presente, permitiendo a las personas migrantes sentirse en casa.

Los huertos interculturales se muestran como espacios de encuen- 
tro y convivencia, no siempre exentos de conflictos, donde se generan procesos de aprendizajes colectivos que favorecen el encuentro multiétnico y la comprensión mutua avanzando hacia la sostenibilidad social de los espacios urbanos. A la vez que se promueve la equidad social de la mano del reconocimiento, la mejora de la autoestima y la aceptación social, se activa el capital social de las personas migrantes (y no migrantes) de la mano de nuevas relaciones interpersonales e institucionales. De esta forma, argumenta el autor, lejos de la opresiva dialéctica de la integración-asimilación, los huertos interculturales permiten dar pasos hacia la inclusión social desde el respeto a la diversidad cultural.

Finalmente, el artículo de Bruno Ronchi, Andrea Amici, Carlo Maía Rossi, Riccardo Primi, Rita Biasi y María Nicolina Ripa, investigadores de Università degli Studi Della Tuscia de Viterbo en Italia, titulado "Multi-temporal analysis of urban and peri-urban land use changes in medieval towns of central Italy", analiza los cambios en los usos del suelo de dos pequeñas ciudades medievales, Vitorchiano y Oriolo Romano, en el centro de Italia, en la provincia de Viterbo en la región de Lazio. Este trabajo tiene un doble objetivo, metodológico y analítico.

Por una parte, en este trabajo se realiza una aportación metodológica para el estudio de los cambios en los usos del suelo y del paisaje. Combinando el uso de la fotografía aérea histórica con el uso de sistemas de información geográfica y el análisis estadístico, se consigue un preciso conocimiento de los cambios en el paisaje y los usos del suelo en las ciudades y en su territorio circundante que se propone como herramienta analítica para la planificación urbanística. El análisis histórico se muestra especialmente relevante, ya que permite identificar los paisajes tradicionales y los usos del suelo en el pasado como parte del patrimonio cultural de las ciudades. Analizar con perspectiva histórica los cambios del paisaje permite comprender los procesos de transformación de una manera más precisa y valorar los usos y paisajes tradicionales que aún hoy se mantienen como valores patrimoniales a conservar.

La metodología propuesta se aplica al análisis de los cambios en el paisaje en las ciudades medievales de Vitorchiano y Oriolo Romano, tanto en los cascos históricos como en el espacio periurbano en esa franja de terreno de encuentro entre lo rural y lo urbano. Se analizan fotografías aéreas y datos de 1934, 1954, 1971, 1989 y 2010, poniendo de manifiesto que los cambios en los usos del suelo se producen mayoritariamente tras 1954 e intensificándose en las décadas posteriores. Los resultados de este trabajo permiten cuantificar con precisión los cambios en los usos del suelo que se caracterizan por un incremento de la urbanización, sobre todo para uso residencial, a costa de la pérdida de los suelos agrícolas, sobre todo los extensivos, y naturales.

Los suelos agrícolas y naturales que todavía existen en el municipio, sobre todo en el espacio periurbano, junto con los espacios verdes y vacantes de las zonas urbanas, son espacios a preservar en la construcción de ciudades sostenibles. La agricultura urbana aparece como un elemento esencial en los paisajes tradicionales de las ciudades medievales y como actividad a mantener y reincorporar en las mismas en la actualidad de cara al futuro. Con esta premisa, este trabajo aporta una herramienta analítica de gran utilidad para la planificación urbana.

En el apartado de Lecturas, Eventos y Debates se publican dos textos. El primero presenta la experiencia del Programa de Agricultura 
Urbana de la ciudad de Rosario en Argentina, que actualmente incluye 67 hectáreas de terreno urbano de las cuales 19 hectáreas son espacios socioproductivos y 48 hectáreas se destinan al ocio y el deporte. Actualmente participan unos 250 huerteros y huerteras aliados con en torno a 400 consumidores estables, además de desarrollar actividades educativas con 40 escuelas. Las verduras se venden tanto a través de "bolsones" entregados a domicilio como en ferias semanales en distintos puntos de la ciudad.

Este programa tiene su origen en la propuesta de una organización social no gubernamental de incorporar la agroecología a la ciudad como estrategia de generación de empleo y sostenibilidad urbana que se plasmó en las primeras huertas urbanas. Esta iniciativa se transformó en 2001 en un amplio movimiento social de respuesta autogestionada frente a la crisis y la exclusión social generada. Poco después, la municipalidad reconoce la importancia de la agricultura urbana como estrategia urbana multifuncional que se transforma en una política pública gubernamental.

En las distintas etapas, el Programa de Agricultura Urbana atiende distintas necesidades y ofrece distintos servicios a la ciudad y a las personas que cultivan estos espacios. En los años de la crisis la emergencia alimentaria centró todos los esfuerzos, pero en los últimos años la agricultura urbana desarrolla las potencialidades de su multifuncionalidad atendiendo necesidades múltiples de diversos grupos ciudadanos, desde el ocio o la educación ambiental a la integración social. En la actualidad, en un contexto de fuerte desigualdad económica pero alejado de la exclusión extrema de los años álgidos de la crisis, el Programa de Agricultura Urbana en Rosario se centra en fortalecer la organización interna de la red de huerteros y huerteras, desarrollar canales cortos de comercialización con la visión del consumo responsable, el comercio justo y la economía solidaria, así como mejorar la dimensión ambiental, paisajística y turística del hábitat urbano en la ciudad de Rosario.

Finalmente, Henk Renting, de la Fundación RUAF, firma el documento titulado "Proyectos de investigación europeos y congresos internacionales sobre agricultura urbana y periurbana", donde presenta los objetivos, enfoques y principales contenidos de distintos proyectos europeos de investigación centrados en la agricultura urbana y periurbana que actualmente se están desarrollando. En concreto se presenta el trabajo que se está realizando en dos acciones COST, orientadas a generar redes europeas de investigación. Una de estas acciones está centrada en la agricultura urbana en general, mientras que la otra es específica para una forma concreta de agricultura urbana, los huertos familiares de ocio. También se presenta el proyecto de investigación SUPURBFOOD "Hacia modos sostenibles de aprovisionamiento alimentario urbano y periurbano" que, a partir de diversos casos de estudio en siete ciudades-región europeas, aborda el análisis de las redes alimentarias que articulan producción y consumo en torno a la ciudad. Finalmente, se presentan las Conferencias Internacionales que anualmente se vienen celebrando desde 2009 a iniciativa del Grupo de Trabajo sobre "Planificación Alimentaria Sostenible" de la Asociación de Escuelas Europeas de Planificación (AESOP). Desde sus inicios, la agricultura urbana y periurbana ha recibido una especial atención en los debates en estas conferencias que se han convertido en un importante espacio de encuentro de investigadores e investigadoras especializadas en este tema. 
El conjunto de las contribuciones que se incluyen en este segundo número monográfico, y que complementan las del número anterior, de la Revista Hábitat y Sociedad aportan tanto evidencias de la intensa innovación social que existe actualmente en torno a la agricultura urbana como ideas y propuestas técnicas y políticas para su incorporación a la planificación y diseño de ciudades con criterios de sostenibilidad y equidad.

SOLER MONTIEL, Marta y RENTING, Henk. La agricultura urbana en la planificación de las ciudades: entre la participación y el mercado. Hábitat y Sociedad, 2014, n. ${ }^{\circ}$, p. p- 5 -11.

$<w w w . h a b i t a t y s o c i e d a d . u s . e s>$

http://dx.doi.org/10.12795/HabitatySociedad.2014.i7.01 